\title{
Incomplete Reiter's syndrome following chemotherapy of acute myeloid leukaemia
}

\author{
Finella Dharmasena ${ }^{1}$, Helen Englert ${ }^{2}$, D. Catovsky ${ }^{1}$, D.A.G. Galton ${ }^{1}$ and H.C. \\ Drysdale $^{3}$
}

\begin{abstract}
${ }^{1}$ Medical Research Council Leukaemia Unit, ${ }^{2}$ Rheumatology Unit, Royal Postgraduate Medical School, Hammersmith Hospital, London W12 0HS and ${ }^{3}$ Department of Haematology, Princess Margaret Hospital, Okus Road, Swindon, UK.
\end{abstract}

\begin{abstract}
Summary: Reiter's syndrome and other reactive arthritides have been described following infection with various organisms although they can occur in unusual circumstances without an obvious infectious precipitant. We have recently witnessed two attacks of reactive arthritis and keratoderma blenorrhagica occurring in an HLA B27 adult male following chemotherapy on two separate occasions with the same drugs for acute myeloid leukaemia. No attacks occurred before or following the cessation of these drugs. This supports the view that in Reiter's syndrome a common pathogenic pathway is triggered by an 'arthritogenic factor' which in this case appears to have been chemical.
\end{abstract}

\section{Introduction}

Reiter's syndrome is the term applied to the classical triad of non-specific urethritis, conjunctivitis and arthritis first described by Reiter in 1916. Other characteristic features of Reiter's syndrome are mucocutaneous lesions such as keratoderma blenorrhagica and circinate balanitis, and Achilles tendinitis and plantar fasciitis which occur in $50 \%$ of patients (Keat et al., 1979). Patients who present with clinical features typical of the syndrome but lack the full diagnostic triad are said to have an incomplete Reiter's syndrome' (Arnett, 1979). The term 'reactive arthritis' was introduced by Aho et al. (1973) to describe arthritis which occasionally follows bacterial infections of the intestinal and urinary tracts but recent reviews offer a wide variety of definitions which include a broad range of infection-associated arthropathies, viral arthritis and the arthritis complicating intestinal by-pass surgery for obesity.

Reiter's syndrome has been described following infection with various organisms, although sometimes no definite infective event is recognized (Csonka, 1979). We have recently witnessed two attacks of reactive arthritis and keratoderma blenorrhagica occurring in an HLA B27 adult man following chemotherapy on two separate occasions with the same drug regimen for acute myeloid leukaemia (AML).

Correspondence: F. Dharmasena, M.B., M.R.C.P.

Accepted: 30 May 1986

\section{Case report}

A 36 year old man was discovered to have AML when he presented with a 5-week history of malaise and mouth ulcers. His white blood count was $28.5 \times 10^{9} / 1$, haemoglobin $5.6 \mathrm{~g} / \mathrm{dl}$ and platelets $192 \times 10^{9} / 1$. Differential count showed $54 \%$ blasts, $20 \%$ monocytes, $8 \%$ neutrophils, $9 \%$ lymphocytes, $4 \%$ metamyelocytes, $4 \%$ myelocytes and $1 \%$ promyelocytes. A bone marrow aspirate confirmed the diagnosis of AML, myelomonocytic type. He had been well previously and had never had attacks suggestive of reactive arthritis or Reiter's syndrome. Examination revealed enlarged cervical, axillary and groin lymph nodes. His spleen was palpable $4 \mathrm{~cm}$ below the costal margin. There were no ocular, joint or skin lesions.

He was treated with daunorubicin $50 \mathrm{mg} / \mathrm{m}^{2}$ i.v. on days 1,3 and 5 , cystosine arabinoside $100 \mathrm{mg} / \mathrm{m}^{2}$ twice daily by i.v. infusion over 24 hours and 6-thioguanine $75 \mathrm{mg} / \mathrm{m}^{2}$ p.o., b.d. from days 1-10 inclusive. Allopurinol $300 \mathrm{mg} /$ day was administered from one day before to 15 days after starting chemotherapy. Eight days after starting treatment he became febrile, but no organisms were grown from serial cultures of urine, stools, throat swabs and blood. Early morning urine smears were negative. The fever responded to treatment with ceftazidime, but recurred. Ceftazidime was replaced by amikacin and piperacillin to which he responded. At this time pigmented patches appeared on his palms and soles which evolved over $3 \frac{1}{2}$ weeks into classical keratoderma blenorrhagica. Subungual

(C) The Fellowship of Postgraduate Medicine, 1986 
hyperkeratoses and painless mouth ulcers were also noted. Left-knee arthritis developed 2 weeks after starting chemotherapy and right-knee arthritis followed 4 days later. Aspiration from both knees yielded large amounts of clear, straw-coloured fluid of low viscosity, without cells, crystals or organisms. Further analysis revealed high complement levels in the fluid when compared to the total serum complement. Tissue typing showed the presence of HLA B27. No features of urethritis or enteritis preceded the development of the arthritis. Serum uric acid was normal. A diagnosis of an incomplete Reiter's syndrome was made, as the full diagnostic triad including urethritis and conjunctivitis was not present. The joint symptoms were treated with piroxicam $20 \mathrm{mg}$ nocte. The mouth ulcers and skin lesions healed in 2 weeks. The joint effusions gradually subsided, leaving a minimal degree of stiffness on movement in both joints. X-rays of the knee joints did not show any erosive changes.

When the blood counts returned to normal levels, he received a second course of the same drugs 6 weeks after the first course. Mouth ulcers and keratoderma blenorrhagica recurred, but the joint effusions did not. Fever recurred and was treated with the same antibiotics as before. No organisms were isolated despite extensive microbial surveillance. Serological tests have not demonstrated infection by Campylobacter, Clostridium difficile, Chlamydia, Yersinia, rubella or any other viruses.

Following his third course of chemotherapy, which consisted of amsacrine, 5'azacytidine and etoposide given intravenously at doses of $100 \mathrm{mg} / \mathrm{m}^{2} /$ day for 5 days, no skin or joint lesions developed although fever recurred. Similarly, his last course of chemotherapy, which was melphalan at a dose of $70 \mathrm{mg} / \mathrm{m}^{2}$ by bolus injection, did not produce any skin or joint lesions though fever recurred. The fever was treated with ceftazidime, piperacillin and amikacin, and amphotericin on both occasions. His clinical course

\section{References}

AHO, K., AHVONEN, P., LASSUS, A., SIEVERS, K. \& TIILIKAINEN, A. (1973). HLA antigen 27 and reactive arthritis. Lancet, ii, 157.

ARNETT, F.C. (1979). Incomplete Reiter's syndrome: clinical comparisons with classical triad. Annals of the Rheumatic Diseases, 38 (Suppl I), 73.

CSONKA, G.W. (1979). Long-term follow-up and prognosis of Reiter's syndrome. Annals of the Rheumatic Diseases, 38 has been characterized by oligoarthritis, mouth ulcers and keratoderma blenorrhagica. At no stage has he developed urethritis, plantar fasciitis, Achilles tendinitis, or ocular symptoms. He is in haematological remission without any joint or skin disease, 10 months from presentation.

\section{Discussion}

Although Reiter's syndrome has been reported following a variety of infections, sometimes no definite infective event is recognized, and the trigger may be non-infective. Our patient had never experienced any symptoms, however mild, suggestive of previous episodes of Reiter's syndrome. The first attack appeared to follow the administration of anti-leukaemic chemotherapy, although a previous infection before admission cannot be ruled out. It is of interest that his second course of chemotherapy produced an attack but the third and fourth courses which consisted of different chemotherapeutic agents did not. In 0 addition, no further attacks have occurred after treatment was stopped. The development of Reiter's syndrome following anti-leukaemic chemotherapy appears not to have been a chance association. The presence of HLA B27 indicated the genetic predisposition which was triggered in this case by a non-infe@ tious agent. This supports the hypothesis that the agents which trigger reactive arthritis share a commor pathway by which they produce the clinical syndrome.

We subsequently reviewed the HLA status of 61 other patients with AML who received the same treatment. Only one 37 year old woman had HLA B27. She did not develop any joint or skin disease following the use of the same agents. It is therefore possible that the development of the syndrome is multifactorial and still to be determined.

(Suppl I), 24.

KEAT, A.C., MAINI, R.N., PEGRUM, G.D. \& SCOTT, J.T. (1979). The clinical features and HLA associations of reactive arthritis associated with non-gonococcal urethritis. Quarterly Journal of Medicine, 48, 323.

REITER, H. (1916). Ueber eine bisher unbekannte Spirochaeteninfektion. Deutsche medizinische Wochenschrift, 42, 1536. 